\title{
MANFREDA BULBULIFERA (AGAVACEAE), ESPECIE NUEVA DE MÉXICO
}

\section{Carlos Castillejos-Cruz y Eloy Solano}

\author{
Universidad Nacional Autónoma de México, Facultad de Estudios Superiores \\ Zaragoza, Herbario FEZA \\ Apdo. postal 9-020, Iztapalapa, 09230 México, D.F. México. \\ carlcasti@colpos.mx
}

\begin{abstract}
RESUMEN
Se describe e ilustra Manfreda bulbulifera, especie nueva para la ciencia, descubierta en Mexcalcingo, municipio de Colotlipa, estado de Guerrero. Esta planta pertenece al grupo de Manfreda guttata, el cual se caracteriza por presentar el tubo del perianto reducido, hojas lineares a lanceoladas y talla pequeña. Muestra similitudes con Manfreda guttata (Jacobi et Bouché) Rose, M. littoralis García-Mend., A. Castañeda et S. Franco y M. rubescens Rose. Difiere de éstas por sus cormos comprimidos, bulbillos en la porción distal del cormo, tubo periantal diminuto, sus segmentos filiformes, estambres y estilo mucho más largos que el tubo y ovario obclaviforme.
\end{abstract}

Palabras clave: Agavaceae, Guerrero, Manfreda, México, taxonomía.

\begin{abstract}
Manfreda bulbulifera, a new species from Mexcalcingo, municipio Colotlipa, Guerrero, Mexico, is described and illustrated. The new species belongs to the group of Manfreda guttata, which is characterized by the presence of a reduced perianth tube, linear to lanceolate leaves and small-sized plants. Manfreda bulbulifera shows similarities with M. guttata (Jacobi et Bouché) Rose, M. littoralis García-Mend., A. Castañeda et S. Franco and $M$. rubescens Rose. The new species differs from the others by its compressed corms, bulblets at the distal portion of the corm, very small perianthal tube, filiform segments, stamens and style longer than the tube and obclavate ovary.
\end{abstract}

Key words: Agavaceae, Guerrero, Manfreda, Mexico, taxonomy. 
Durante la revisión sistemática del género Manfreda, se observaron dos ejemplares, uno recolectado por H. D. Irby y C. M. Rowell Jr. y el otro por G. F. Rhymes y C. M. Rowell Jr., con números de recolecta 3571 y 3855 respectivamente, ambos depositados en el herbario MICH. Dichos especímenes provienen del estado de Guerrero, ocho millas al sureste de Colotlipa. Esta localidad la exploramos en el año 2006 durante nuestros recorridos por toda el área de distribución del género en México. Los ejemplares recolectados mostraron características morfológicas que difieren ampliamente de las especies anteriormente descritas; consecuentemente se propone como nueva a:

Manfreda bulbulifera Castillejos \& E. Solano, sp. nov. Fig. 1.

Herba perennis; cormus 5-7 mm longus, compressus, bulbulis $0.5-1.5 \mathrm{~cm}$ longis circumdatus; inflorescentiae pars fertilis 7.0-8.5(-12.0) $\mathrm{cm}$ longa, laxa, 7-10 floribus, perianthii segmentis linearibus, 2.5-3.0(-3.5) cm longis, 1.5-3.0 mm latis; ovarium $1.2 \mathrm{~cm}$ longum, $3.2 \mathrm{~mm}$ latum, obclavatum; perianthii tubus minutus $0.5-1$ $\mathrm{mm}$ longus, 2-3 mm latus, ad apicem ovarii sine constrictione.

Planta herbácea, perenne. Cormo de 5-7 mm de largo, con raíces contráctiles carnosas y fibrosas, comprimido, en su porción distal con bulbillos de $0.5-1.5 \mathrm{~cm}$ de largo, provistos de uno o dos catafilos, bulbo de 3-5 cm de largo, 2.5-3.0 cm de diámetro en la base, ovoide, cubierto por las bases de las hojas secas, éstas de 4.5$6.0 \mathrm{~cm}$ de largo, membranáceas en la base y con la parte superior fibrosa. Hojas 2 a 4, de 12-25 cm de largo, 3-6 mm de ancho, lineares a lanceoladas, atenuadas, acanaladas, postradas o semierectas, verdes-amarillentas a verdes oscuras, glabras, a menudo ligeramente verrugosas, margen hialino, angosto, entero, al microscopio en algunas secciones finamente papiloso, ápice agudo. Inflorescencia de 70-80(-86) $\mathrm{cm}$ de largo, erecta, porción fértil de 7.0-8.5(-12.0) cm de largo, laxa, con 7-10 flores, eje verdoso a glauco en toda su longitud, brácteas 5-7, con la base truncada y algo inclinada, las inferiores lineares, las superiores deltoides, la tercera bráctea basal de 3.5-6.0(-7.2) $\mathrm{cm}$ de largo, 2-3 $\mathrm{mm}$ de ancho, verde-amarillenta, brácteas florales de 4-6 mm de largo, 1-2 mm de ancho. Flores de 2.5-3.2 cm de largo, difusas, sobre pedicelos de 1-3 mm, verdes-amarillentas, a verdes-blanquecinas; tubo del perianto diminuto, de 0.5-1.0 mm de largo, 2-3 mm de ancho, sin constricción en el ápice del ovario, segmentos de 2.5-3.0(-3.5) cm de largo, 1.5-3.0 mm de ancho, lineares, erectos o reflexos, ápice semisuculento, cuculado, con un mechón de tricomas blancos; los filamentos exceden al tubo por 5.0-6.0 cm, adnados a la boca del tubo, libres en 



Fig. 1. Manfreda bulbulifera. A. planta con inflorescencia; B. bulbo con bulbillos; C. fruto; D. flor disecada; E. semilla. Ilustración basada en los ejemplares de Castillejos et al. 1807 (FEZA, MEXU). 
la base de los lóbulos, dispuestos en un mismo nivel, erectos en la antesis, rojizos; anteras de 7-8(-9) $\mathrm{mm}$ de largo, 1.0-1.5 $\mathrm{mm}$ de ancho, verdes-amarillentas a verdesrojizas; ovario de $12 \mathrm{~mm}$ de largo, $3.2 \mathrm{~mm}$ de ancho, obclaviforme, verde-amarillento; estilo de 5.0 a $6.8 \mathrm{~cm}$ de largo, estigma trilobado, verde-amarillento. Fruto capsular, de 1.0-1.2 cm de largo, 1.1-1.2 cm de diámetro, subgloboso, triquetro, ápice sin cicatriz. Semillas de 3-4 mm de largo, $3 \mathrm{~mm}$ de ancho, plano-cóncavas, negras, opacas.

Tipo. México, Guerrero: municipio de Colotlipa, camino de terracería al SE de Colotlipa, entre El Epazote y Mexcalcingo, 1258 m, bosque de pino-encino perturbado, 8 julio 2006, C. Castillejos et al. 1807 (holotipo: MEXU; isotipos: CHAPA, FEZA, IEB, MICH, NY, US).

Paratipos. México, Guerrero: 8 mi SE Colotlipa, G. F. Rhymes \& C. M. Rowell Jr. 3855 (MICH); 8 mi SE Colotlipa, H. D. Irby \& C. M. Rowell Jr. 3571 (MICH).

Fenología. Florece de junio a julio y fructifica en agosto.

Hábitat y distribución. Manfreda bulbulifera crece en sitios con pendiente ligera, algo rocosos, donde se acumula suelo rico en materia orgánica y de textura arenosa, dentro de bosques de pino-encino, en altitudes de 1200 a $1260 \mathrm{~m}$. Es una planta que forma poblaciones densas y localizadas. Aparentemente endémica de la localidad tipo. Por su distribución restringida y la alteración de su hábitat esta especie pudiera catalogarse como amenazada. El epíteto específico alude a la presencia de bulbillos en el cormo.

Por sus características florales y foliares Manfreda bulbulifera pertenece al grupo de M. guttata reconocido por Verhoek-Williams (1975). En este conjunto las hojas son delgadas a semisuculentas, con el margen foliar hialino, liso o áspero, papiloso o eroso-denticulado al microscopio; el ovario se proyecta ligeramente dentro del tubo y provoca que el perianto persista en las cápsulas y deje una cicatriz anular en la parte apical de las mismas. Las especies reunidas por Verhoek-Williams (1975) en este grupo son: Manfreda guttata (Jacobi et Bouché) Rose, M. planifolia (S. Watson) Rose, M. pringlei Rose y M. rubescens Rose, y según García Mendoza et al. (2000) y Castañeda et al. (2005), M. fusca Ravenna, M. galvaniae A. Castañeda, S. Franco et García-Mend., así como M. littoralis García-Mend., A. Castañeda et S. Franco, también forman parte del mismo. La mayoría de las mencionadas espe- 
Cuadro 1. Características comparativas de Manfreda bulbulifera y especies afines. Los datos de M. guttata corresponden a Verhoek-Williams (1975); los de M. rubescens a McVaugh (1989) y los de M. littoralis provienen de García-Mendoza et al. (2000), todos ellos fueron enriquecidos con observaciones propias.

\begin{tabular}{|c|c|c|c|c|}
\hline & M. bulbulifera & M. guttata & M. rubescens & M. littoralis \\
\hline Cormo & $\begin{array}{l}\text { comprimido, } \\
\text { 5-7 mm de } \\
\text { largo, bulbillos } \\
\text { presentes }\end{array}$ & \begin{tabular}{|l|} 
ovoide, \\
$1.8-4$ x $1.3-3 \mathrm{~cm}$, \\
rizomatoso
\end{tabular} & $\begin{array}{l}\text { cilíndrico, } \\
1.5-4 \text { x } 1-1.8 \mathrm{~cm}\end{array}$ & $\begin{array}{l}\text { cónico- truncado, } \\
3-5 \times 2.5-3 \mathrm{~cm}, \\
\text { rizomatoso }\end{array}$ \\
\hline $\begin{array}{l}\text { Bases secas } \\
\text { de las hojas }\end{array}$ & $\begin{array}{l}\text { fibrosas, } 4.5-6 \\
\mathrm{~cm} \text { de largo }\end{array}$ & $\begin{array}{l}\text { fibrosas, } 3-6 \mathrm{~cm} \\
\text { de largo }\end{array}$ & $\begin{array}{l}\text { fibrosas, } 0.5 \mathrm{~cm} \\
\text { de largo }\end{array}$ & $\begin{array}{l}\text { membranáceas, } \\
3-5 \text { de largo y } \\
2.5 \mathrm{~cm} \text { de ancho }\end{array}$ \\
\hline Hojas & $\begin{array}{l}2-4,12-25 \mathrm{~cm} \mathrm{x} \\
3-6 \mathrm{~mm}, \text { lineares } \\
\text { a linear-lanceola- } \\
\text { das, atenuadas }\end{array}$ & $\begin{array}{l}\text { 2-7(-8), 15-38 } \\
\text { x 1.3-3.1 cm, } \\
\text { linear-lanceola- } \\
\text { das, atenuadas }\end{array}$ & $\begin{array}{l}3-6(-10), 30-60 \\
x \text { 0.6-1.4 cm, } \\
\text { lineares, } \\
\text { truncadas }\end{array}$ & $\begin{array}{l}\text { 4-6, 9-20(-27) } \\
\text { x } 2.58 .6) \mathrm{cm}, \\
\text { elípticas a elípti- } \\
\text { co-lanceoladas, } \\
\text { atenuadas y } \\
\text { pseudopecioladas }\end{array}$ \\
\hline $\begin{array}{l}\text { Superficie } \\
\text { de las hojas }\end{array}$ & $\begin{array}{l}\text { glabra a ligera- } \\
\text { mente verrugosa } \\
\text { en el haz }\end{array}$ & $\begin{array}{l}\text { ligeramente } \\
\text { verrugosa } \\
\text { en el haz }\end{array}$ & $\begin{array}{l}\text { haz glabro, } \\
\text { envés papiloso } \\
\text { sobre las } \\
\text { nervaduras }\end{array}$ & $\begin{array}{l}\text { finamente papi- } \\
\text { losa }\end{array}$ \\
\hline Margen & $\begin{array}{l}\text { hialino, irre- } \\
\text { gularmente } \\
\text { papiloso }\end{array}$ & $\begin{array}{l}\text { hialino, eroso- } \\
\text { denticulado }\end{array}$ & hialino, entero & $\begin{array}{l}\text { hialino, finamente } \\
\text { papiloso en } \\
\text { algunas secciones }\end{array}$ \\
\hline $\begin{array}{l}\text { Eje de la in- } \\
\text { florescencia }\end{array}$ & $\begin{array}{l}70-80(-86) \mathrm{cm} \\
\text { de largo }\end{array}$ & $\begin{array}{l}(61-) 90-156 \mathrm{~cm} \\
\text { de largo }\end{array}$ & $\begin{array}{l}60-85(-135) \mathrm{cm} \\
\text { de largo }\end{array}$ & $\begin{array}{l}60-70(-80) \mathrm{cm} \mathrm{de} \\
\text { largo }\end{array}$ \\
\hline $\begin{array}{l}\text { Porción fértil } \\
\text { inflorescencia }\end{array}$ & $\begin{array}{l}\text { laxa, } 7.0-8.5 \\
(-12.0) \mathrm{cm}\end{array}$ & \begin{tabular}{|l|} 
densa en la \\
porción distal \\
$(2.3-) 8-14 \mathrm{~cm}$ \\
\end{tabular} & $\begin{array}{l}\text { densa, 6-8 } \\
(-18) \mathrm{cm}\end{array}$ & $\begin{array}{l}\text { densa } 3.5-4.5 \\
(-6.5) \mathrm{cm}\end{array}$ \\
\hline $\begin{array}{l}\text { Número de } \\
\text { flores por } \\
\text { inflorescencia }\end{array}$ & $7-10$ & $20-30$ & $5-9(-16)$ & $8-12$ \\
\hline $\begin{array}{l}\text { Color y fra- } \\
\text { gancia de las } \\
\text { flores }\end{array}$ & $\begin{array}{l}\text { verde-amari- } \\
\text { llento a verde- } \\
\text { blanquecino, sin } \\
\text { fragancia }\end{array}$ & $\begin{array}{l}\text { verde-amari- } \\
\text { llento con tintes } \\
\text { púrpura, olor a } \\
\text { cebolla }\end{array}$ & $\begin{array}{l}\text { púrpura-oscuro, } \\
\text { a verde-pardus- } \\
\text { co, sin fragancia }\end{array}$ & $\begin{array}{l}\text { verde-amarillen- } \\
\text { to, glaucas, olor } \\
\text { acre }\end{array}$ \\
\hline
\end{tabular}


Cuadro 1. Características comparativas de Manfreda bulbulifera y especies afines. Continuación.

\begin{tabular}{|c|c|c|c|c|}
\hline & M. bulbulifera & M. guttata & M. rubescens & M. littoralis \\
\hline $\begin{array}{l}\text { Largo del } \\
\text { tubo periantal }\end{array}$ & $0.5-1.0 \mathrm{~mm}$ & $0.3-1.2 \mathrm{~cm}$ & $3-5(-6) \mathrm{mm}$ & $1-2(-2.8) \mathrm{mm}$ \\
\hline $\begin{array}{l}\text { Segmentos } \\
\text { del perianto }\end{array}$ & $\begin{array}{l}2.5-3.0(-3.5) \\
x \quad 0.15-0.3 \mathrm{~cm}, \\
\text { lineares, erectos } \\
\text { o reflexos }\end{array}$ & $\begin{array}{l}(0.6-) 1-1.5 \mathrm{x} \\
0.2-0.3 \mathrm{~cm}, \\
\text { lineares reflexos } \\
\text { a extendidos }\end{array}$ & $\begin{array}{l}1.2 \times 0.2-0.35 \\
\mathrm{~cm} \text {, elípticos } \\
\text { a oblongos, } \\
\text { reflexos }\end{array}$ & \begin{tabular}{|l|}
$0.9-1(-1.1) \mathrm{x}$ \\
$0.15-0.2(-0.35)$ \\
$\mathrm{cm}$, oblongos, \\
erectos o reflexos
\end{tabular} \\
\hline Filamentos & $5-6 \mathrm{~cm}$ & $2-2.9(-4.1) \mathrm{cm}$ & $1.6-2.5 \mathrm{~cm}$ & $(1-) 1.4-1.8 \mathrm{~cm}$ \\
\hline $\begin{array}{l}\text { Inserción de } \\
\text { los filamen- } \\
\text { tos }\end{array}$ & $\begin{array}{l}\text { en la boca del } \\
\text { tubo }\end{array}$ & $\begin{array}{l}\text { en la boca del } \\
\text { tubo }\end{array}$ & 3/4 del tubo & $\begin{array}{l}\text { en la boca del } \\
\text { tubo }\end{array}$ \\
\hline Ovario & $\begin{array}{l}12 \times 3.2 \mathrm{~mm}, \\
\text { obclavado }\end{array}$ & $\begin{array}{l}\text { 6-12(-15) x 3-4 } \\
\text { mm, cilíndrico }\end{array}$ & $\begin{array}{l}8 \times 4 \mathrm{~mm} \text {, cilín- } \\
\text { drico }\end{array}$ & $\begin{array}{l}3-5(-6) \times 1.52 .5 \\
(-4) \mathrm{mm}, \\
\text { cilíndrico }\end{array}$ \\
\hline Fruto & $\begin{array}{l}1.0-1.2 \times 1.1-1.2 \\
\mathrm{~cm}, \text { subgloboso, } \\
\text { triquetro, ápice } \\
\text { sin cicatriz }\end{array}$ & $\begin{array}{l}1.6-2.4 \text { x 1-1.9 } \\
\mathrm{cm} \text {, ovoide, ápi- } \\
\text { ce con cicatriz }\end{array}$ & $\begin{array}{l}0.7-1 \times 0.7-0.8 \\
\mathrm{~cm} \text {, elipsoidal, } \\
\text { ápice sin cicatriz }\end{array}$ & $\begin{array}{l}0.9-1 \times 0.8-1 \mathrm{~cm}, \\
\text { subgloboso, ápice } \\
\text { con cicatriz }\end{array}$ \\
\hline $\begin{array}{l}\text { Hábitat, } \\
\text { altitud y dis- } \\
\text { tribución }\end{array}$ & $\begin{array}{l}\text { bosque de pino- } \\
\text { encino, } 1200 \text { a } \\
1260 \mathrm{~m} \text {. Hasta } \\
\text { ahora únicamen- } \\
\text { te conocida de la } \\
\text { localidad tipo }\end{array}$ & $\begin{array}{l}\text { pastizales, } \\
\text { sitios abiertos } \\
\text { o rocosos, en } \\
\text { matorrales, } 1800 \\
\text { a } 2400 \text { m. } \\
\text { Ags., Chih., } \\
\text { Dgo., Jal., Gto., } \\
\text { Qro., S.L.P., Zac. }\end{array}$ & $\begin{array}{l}\text { bosque de } \\
\text { encino, bosque } \\
\text { tropical caduci- } \\
\text { folio, } 1000 \mathrm{~m} \text {. } \\
\text { Nay., Jal. }\end{array}$ & $\begin{array}{l}\text { selva mediana } \\
\text { subcaducifolia y } \\
\text { baja caducifolia; } \\
20-260 \text { m. } \\
\text { Gro., Oax. }\end{array}$ \\
\hline
\end{tabular}

cies son endémicas de México, excepto $M$. fusca, que habita en los alrededores de Chimaltenango y Comalapa, Guatemala. M. bulbulifera muestra similitudes en su morfología con M. guttata, M. littoralis y M. rubescens, de las que se diferencia por las características mostradas en el Cuadro 1.

Manfreda bulbulifera se distingue por su cormo comprimido y presencia de bulbillos que funcionan en la propagación vegetativa, rasgos hasta ahora sólo conocidos en esta especie; tubo periantal diminuto, segmentos filiformes, estambres y estilo muy largos en relación con el tubo del perianto y ovario obclaviforme. Tal forma peculiar del ovario es rara en este género, únicamente se comparte con M. sileri 
Verh.-Will. y M. variegata (Jacobi) Rose, aunque en las descripciones de las mismas, esta característica no había sido señalada. Cabe mencionar que Thiede (2001), con base en estudios cladísticos y caracteres moleculares realizados por Bogler y Simpson (1996) y Eguiarte et al. (2000), considera a Manfreda como un subgénero de Agave. Sin embargo, preferimos mantener a Manfreda como género separado, ya que en los estudios filogenéticos realizados se incluyen pocas especies de cada género.

\section{AGRADECIMIENTOS}

Se agradece a Fernando Chiang y Abisaí García Mendoza, por la revisión crítica del manuscrito, el primero elaboró la diagnosis en latín. Ramiro Ríos G., ha recorrido con nosotros casi toda el área de distribución en México del género Manfreda. Este proyecto ha sido financiado por DGAPA-PAPIIT, UNAM, convenio IN211103. El curador del herbario MICH facilitó el préstamo de ejemplares.

\section{LITERATURA CITADA}

Bogler, D. J. y B. B. Simpson. 1996. Phylogeny of Agavaceae based on ITS RDNA sequence variation. Amer. J. Bot. 83: 1225-1235.

Eguiarte, L. E., V. Souza y A. Silva-Montellano. 2000. Evolución de la familia Agavaceae: filogenia, biología reproductiva y genética de poblaciones. Bol. Soc. Bot. Méx. 66: $131-150$.

Castañeda, A., I. S. Franco y A. García-Mendoza. 2005. Manfreda galvaniae (Agavaceae), especie nueva de México, con notas sobre la ubicación taxonómica de $M$. malinaltenangensis Matuda y su lectotipificación. Acta Bot. Mex. 72: 65-76.

García-Mendoza, A., A. Castañeda e I. S. Franco. 2000. Manfreda littoralis (Agavaceae), nueva especie de Guerrero y Oaxaca, México. Acta Bot. Mex. 50: 39-45.

McVaugh, R. 1989. Liliaceae. Flora Novo-Galiciana. 15: 227-237.

Thiede, J. 2001. Agavaceae. In: Eggli, U. (ed.). Illustrated handbook of succulent plants: monocotyledons. Springer Verlag. Nueva York. pp. 5-76.

Verhoek-Williams, S. E. 1975. A study of the tribe Poliantheae (including Manfreda) and revisions of Manfreda and Prochnyanthes (Agavaceae). Tesis doctoral. Cornell University. Ithaca, Nueva York. 405 pp. 
